\title{
FOURIER SUMMATION WITH KERNELS DEFINED BY JACOBI POLYNOMIALS
}

\author{
R. LASSER
}

(Communicated by J. Marshall Ash)

\begin{abstract}
Trigonometric polynomial kernels defined by Jacobi polynomials are investigated. They generalize the classical Dirichlet kernel and the Fejér kernel. The asymptotic behavior of the corresponding Fourier summation obtained is leading to optimal kernels.
\end{abstract}

The Dirichlet kernel and the Fejér kernel are basic tools in the theory of classical Fourier expansions. We introduce trigonometric polynomial kernels defined by Jacobi polynomials. One class consists of kernels with alternating signs containing the Dirichlet kernel as a special case; the other class consists of nonnegative kernels containing the Fejér kernel as a special case. Therefore, these kernels are certain parameterized generalizations of the Dirichlet kernel resp. Fejér kernel. We investigate their asymptotic behavior, finding in this way optimal kernels, where optimality is defined with respect to the generalized Lipschitz condition. Throughout this work our main reference for notation and results concerning Fourier series is [4]. For other uses of Jacobi polynomials as summability kernels we refer to $[2,6]$.

\section{Generalizations of THe Dirichlet Kernel}

Fix $\lambda>0$. We study the following row-finite $\theta$ factors, which are strongly related to certain Jacobi polynomials: For $n \in \mathbb{N}_{0}, k=0, \ldots, n$, set

$$
a_{n, k}^{\lambda}=\frac{(\lambda)_{n+k}(\lambda)_{n-k}(n !)^{2}}{(n+k) !(n-k) !\left((\lambda)_{n}\right)^{2}} \text { and } a_{n,-k}^{\lambda}=a_{n, k}^{\lambda} \text {, }
$$

and for $t \in]-\pi, \pi]$, denote

$$
D_{n}^{\lambda}\left(e^{i t}\right)=\sum_{k=-n}^{n} a_{n, k}^{\lambda} e^{i k t} .
$$

For $\lambda=1$ we have the classical Dirichlet kernel.

Proposition 1. We have

$$
D_{n}^{\lambda}\left(e^{i t}\right)=b_{n}^{\lambda} R_{n}^{(\lambda-1 / 2,1 / 2)}(\cos t),
$$

Received by the editors May 30, 1990 and, in revised form, October 1, 1990. 1980 Mathematics Subject Classification (1985 Revision). Primary 42A10.

Key words and phrases. Fourier approximation, Jacobi polynomials.

(C) 1992 American Mathematical Society $0002-9939 / 92 \$ 1.00+\$ .25$ per page 
where $R_{n}^{(\lambda, 1 / 2,-1 / 2)}(x)$ is the $n$th Jacobi polynomial to the parameters $\alpha=\lambda-\frac{1}{2}$, $\beta=-\frac{1}{2}$, see [7], normalized such that $R_{n}^{(\lambda-1 / 2,-1 / 2)}(1)=1$, and where

$$
b_{n}^{\lambda}=\frac{(n !)^{2}(2 \lambda)_{2 n}}{\left.\left((\lambda)_{n}\right)^{2}(2 n) !\right)}
$$

Proof. Formulas (2.7), (3.15), and Gegenbauer's formula (7.5) in [3] yield for $\lambda>-\frac{1}{2}$ :

$$
\begin{aligned}
R_{2 n}^{(\lambda-1 / 2, \lambda-1 / 2)}\left(\cos \frac{t}{2}\right) & =\frac{(2 n) !}{(2 \lambda)_{2 n}} \sum_{k=0}^{2 n} \frac{(\lambda)_{2 n-k}(\lambda)_{k}}{(2 n-k) ! k !} \cos (n-k) t \\
& =\frac{(2 n) !}{(2 \lambda)_{2 n}} \sum_{k=-n}^{n} \frac{(\lambda)_{n+k}(\lambda)_{n-k}}{(n+k) !(n-k) !} e^{i k t}
\end{aligned}
$$

Using (3.13) in [3], we have

$$
R_{n}^{(\lambda-1 / 2,-1 / 2)}(\cos t)=\frac{(2 n) !}{(2 \lambda)_{2 n}} \sum_{k=-n}^{n} \frac{(\lambda)_{n+k}(\lambda)_{n-k}}{(n+k) !(n-k) !} e^{i k t},
$$

which gives identity (3).

Remark. We have chosen the normalization $R_{n}^{(\lambda-1 / 2,-1 / 2)}(1)=1$, since for $\lambda>0$ it follows that $\left|R_{n}^{(\lambda-1 / 2,-1 / 2)}(x)\right| \leq 1$ for every $x \in[-1,1]$, see [7, (7.33.1)]. Hence, a uniform bound for $\left|D_{n}^{\lambda}\left(e^{i t}\right)\right|$ is $b_{n}^{\lambda}$.

Theorem 1. For $\lambda>1$ we have

(i) $\lim _{n \rightarrow \infty} a_{n, k}^{\lambda}=1$ for all $k \in \mathbb{N}_{0}$ and

(ii) $\left\|D_{n}^{\lambda}\right\|_{1} \leq M$ for all $n \in \mathbb{N}_{0}$.

Proof. Statement (i) follows from the asymptotic properties of the Gamma function. In fact, we have

$$
a_{n, k}^{\lambda}=\frac{\Gamma(\lambda+n+k) \Gamma(\lambda+n-k)(\Gamma(1+n))^{2}}{\Gamma(1+n+k) \Gamma(1+n-k)(\Gamma(\lambda+n))^{2}} .
$$

Hence by $[1,6.1 .46]$, we obtain for fixed $k \in \mathbb{N}_{0}$

$$
\lim _{n \rightarrow \infty} a_{n, k}^{\lambda}=\lim _{n \rightarrow \infty} \frac{(n+k)^{\lambda}(n-k)^{\lambda} n^{2}}{(n+k)(n-k) n^{2 \lambda}}=1 .
$$

Statement (ii) follows from asymptotic properties of Jacobi polynomials. Note that

$$
\left\|D_{n}^{\lambda}\right\|_{1}=\frac{b_{n}^{\lambda}}{\pi} \int_{0}^{\pi}\left|R_{n}^{(\lambda-1 / 2,-1 / 2)}(\cos t)\right| d t .
$$

By [7, Theorem 8.21.13], we have for $t \in\left[\frac{1}{n}, \pi-\frac{1}{n}\right]$

$$
R_{n}^{(\lambda-1 / 2,-1 / 2)}(\cos t)=\frac{n !}{(\lambda+1 / 2)_{n} n^{1 / 2}} k(t)\left(\cos (N t+\gamma)+(n \sin t)^{-1} O(1)\right)
$$

where $N=n+\lambda / 2, \gamma=-\lambda \pi / 2$ and

$$
k(t)=\frac{1}{\pi^{1 / 2}} \frac{1}{(\sin t / 2)^{\lambda}} .
$$


Using $[1,6.1 .46]$ again

$$
\lim _{n \rightarrow \infty} \frac{1}{n} b_{n}^{\lambda}=\frac{(\Gamma(\lambda))^{2}}{\Gamma(2 \lambda)} 2^{2 \lambda-1}
$$

follows. Hence, integrating over $\left[0, \frac{1}{n}\right],\left[\frac{1}{n}, \pi-\frac{1}{n}\right]$ and $\left[\pi-\frac{1}{n}, \pi\right]$, we obtain (6)

$\left\|D_{n}^{\lambda}\right\|_{1} \leq M_{1}+M_{2} \frac{b_{n}^{\lambda} n !}{(\lambda+1 / 2)_{n} n^{1 / 2}} \int_{1 / n}^{\pi-1 / n} \frac{d t}{(\sin t / 2)^{\lambda}} \leq M_{1}+M_{3} \frac{1}{n^{\lambda-1}} \int_{1 / n}^{\pi-1 / n} \frac{d t}{t^{\lambda}}$, with constants $M_{1}, M_{2}, M_{3} \geq 0$ not depending on $n$, and (ii) follows.

As is known, Theorem 1 yields (cf. [4, Proposition 1.3.3]) the following result.

Corollary 1. Let $\lambda>1$. Denoting by $X$ one of the Banach spaces $L^{p}(T)$, $1 \leq p<\infty$, or $C(T)$, we have for each $f \in X$ :

(iii) $\lim _{n \rightarrow \infty}\left\|D_{n}^{\lambda} * f-f\right\|_{X}=0$.

Remark. For $X=L^{1}(T)$ and $X=C(T)$ condition (iii) also implies property (i) and (ii), cf. [4, Theorem 1.3.5].

Further we can derive that $\left(D_{n}^{\lambda}\right)_{n \in \mathbb{N}_{0}}$ is saturated in $X$ with order $O\left(n^{-2}\right)$. In fact, by $\Gamma(n+a) / \Gamma(n+b)=n^{a-b}\left(1+c_{1} / n+c_{2} / n^{2}+\cdots\right)$ (compare [1, 6.1.47]), we obtain

$$
\lim _{n \rightarrow \infty} \frac{a_{n, k}^{\lambda}-1}{n^{-2}}=-(\lambda-1) k^{2} .
$$

In particular, Proposition 12.1.2 of [4] implies

Corollary 2. Let $\lambda>1$. If $\left\|D_{n}^{\lambda} * f-f\right\|_{X}=o\left(n^{-2}\right)$ with $n \rightarrow \infty$, then $f$ is constant almost everywhere.

Next we study the order of approximation by $D_{n}^{\lambda} * f$ in comparison with the order of best approximation. Integrating from 0 to $\frac{1}{n}$, we have for $\alpha>0$

$$
\int_{0}^{1 / n} t^{\alpha}\left|D_{n}^{\lambda}\left(e^{i t}\right)\right| d t \leq M b_{n}^{\lambda} \int_{0}^{1 / n} t^{\alpha} d t=O\left(n^{-\alpha}\right) .
$$

In the same way,

$$
\int_{\pi-1 / n}^{\pi} t^{\alpha}\left|D_{n}^{\lambda}\left(e^{i t}\right)\right| d t=O\left(n^{-\alpha}\right)
$$

holds with $n \rightarrow \infty$. According to (6),

$$
\int_{1 / n}^{\pi-1 / n} t^{\alpha}\left|D_{n}^{\lambda}\left(e^{i t}\right)\right| d t \leq M \frac{1}{n^{\lambda-1}} \int_{1 / n}^{\pi-1 / n} t^{\alpha} \frac{1}{t^{\lambda}} d t .
$$

Hence,

$$
\int_{1 / n}^{\pi-1 / n} t^{\alpha}\left|D_{n}^{\lambda}\left(e^{i t}\right)\right| d t= \begin{cases}O\left(n^{-\alpha}\right) & \text { for } 0<\alpha<\lambda-1, \\ O\left(n^{-(\lambda-1)} \ln n\right) & \text { for } \alpha=\lambda-1, \\ O\left(n^{-(\lambda-1)}\right) & \text { for } \alpha>\lambda-1 .\end{cases}
$$

Combining this with [4, Proposition 1.6.3], we obtain 
Corollary 3. Let $\lambda>1$. If $f \in \operatorname{Lip}^{*}(X ; \alpha)$, it follows that

$$
\left\|D_{n}^{\lambda} * f-f\right\|_{X}= \begin{cases}O\left(n^{-\alpha}\right) & \text { for } 0<\alpha<\lambda-1, \\ O\left(n^{-(\lambda-1)} \ln n\right) & \text { for } \alpha=\lambda-1, \\ O\left(n^{-(\lambda-1)}\right) & \text { for } \alpha>\lambda-1 .\end{cases}
$$

Therefore, $D_{n}^{\lambda} * f$ behave for $\lambda>3$ asymptotically as does the element of best approximation (cf. [4, Corollary 2.2.2]).

\section{Generalizations of the Fejér Kernel}

In this section we consider positive sums of the trigonometric polynomials $A_{k}^{\lambda}\left(e^{i t}\right)$. First note that

$$
D_{n}^{\lambda}\left(e^{i t}\right)=\frac{n !}{(\lambda)_{n}} \frac{P_{n}^{(\lambda-1 / 2,-1 / 2)}(\cos t)}{P_{n}^{(-1 / 2, \lambda-1 / 2)}(1)},
$$

where $P_{n}^{(\alpha, \beta)}(x)$ denotes $n$th Jacobi polynomial with the usual normalization $P_{n}^{(\alpha, \beta)}(1)=\frac{(\alpha+1)_{n}}{n !}$.

Fix $\lambda>1$ and $0 \leq \mu \leq \lambda-1$. For $n \in \mathbb{N}_{0}, k=0, \ldots, n$, set

$$
b_{n, k}^{\lambda, \mu}=\frac{(\mu+1)_{n-k}(\mu+1)_{k}(\lambda)_{k}}{(n-k) ! k ! k !} .
$$

Further, set $\beta_{n}^{\lambda, \mu}=\sum_{k=0}^{n} b_{n, k}^{\lambda, \mu}$ and denote

$$
F_{n}^{\lambda, \mu}\left(e^{i t}\right)=\frac{1}{\beta_{n}^{\lambda, \mu}} \sum_{k=0}^{n} b_{n, k}^{\lambda, \mu} D_{k}^{\lambda}\left(e^{i t}\right) .
$$

Now using a profound result by Gasper [5] implies that $F_{n}^{\lambda, \mu}\left(e^{i t}\right) \geq 0$ for all $t \in]-\pi, \pi]$. Note that for $\lambda=1$ and $\mu=0$, we have the classical Fejér kernel. Since for $\lambda>1$ and $0 \leq \mu \leq \lambda-1$ the kernel functions $F_{n}^{\lambda, \mu}$ are positive, we investigate their first Fourier coefficients. From (8), (9) and (1), (2) we obtain

$$
\left(F_{n}^{\lambda, \mu}\right)^{\sim}(1)=\frac{1}{\beta_{n}^{\lambda, \mu}} \sum_{k=0}^{n} b_{n, k}^{\lambda, \mu} \frac{k(k+\lambda)}{(k+1)(k+\lambda-1)} .
$$

We prove now that $\lim _{n \rightarrow \infty}\left(F_{n}^{\lambda, \mu}\right) \wedge(1)=1$ is valid. In fact, given $\varepsilon>0$, we choose $n_{0} \in \mathbb{N}$ such that $1-\frac{k(k+\lambda)}{(k+1)(k+\lambda-1)}<\varepsilon$ for each $k>n_{0}$. Thus, for $n \geq n_{0}$

$$
\begin{aligned}
\left|1-\left(F_{n}^{\lambda, \mu}\right)^{\sim}(1)\right| & \leq \frac{1}{\beta_{n}^{\lambda, \mu}} \sum_{k=0}^{n_{0}} b_{n, k}^{\lambda, \mu}\left(1-\frac{k(k+\lambda)}{(k+1)(k+\lambda-1)}\right)+\varepsilon \\
& \leq \frac{1}{\beta_{n}^{\lambda, \mu}} \sum_{k=0}^{n_{0}} b_{n, k}^{\lambda, \mu}+\varepsilon .
\end{aligned}
$$

Therefore, we have to show that the $\left(1 / \beta_{n}^{\lambda, \mu}\right) \sum_{k=0}^{n_{0}} b_{n, k}^{\lambda, \mu}$ tends to zero with $n \rightarrow \infty$. The asymptotic of the Gamma function yields

$$
\sum_{k=0}^{n_{0}} b_{n, k}^{\lambda, \mu}=O\left(n^{\mu}\right) \text { for } n \rightarrow \infty .
$$


To determine the asymptotic order of $\beta_{n}^{\lambda, \mu}$ we prove the following auxiliary result, which is likely to be known.

Lemma 1. Assume that $u_{k}$ and $v_{k}, k \in \mathbb{N}_{0}$, are two numerical sequences with $u_{k}=O\left(k^{\alpha}\right)$ and $v_{k}=O\left(k^{\beta}\right)$ for $k \rightarrow \infty$ with $\alpha, \beta>-1$. Then, defining

$$
w_{n}=\sum_{k=0}^{n} u_{n-k} v_{k}
$$

we have $w_{n}=O\left(n^{\alpha+\beta+1}\right)$ for $n \rightarrow \infty$.

Proof. Let $\left|u_{k}\right| \leq M_{1} k^{\alpha}$ and $\left|v_{k}\right| \leq M_{2} k^{\beta}$ for any $k \in \mathbb{N}_{0}$. Then

$$
\begin{aligned}
\left|\frac{w_{n}}{n^{\alpha+\beta+1}}\right| \leq & \frac{M_{1} M_{2}}{n^{\alpha+\beta+1}} \sum_{k=1}^{n-1}(n-k)^{\alpha} k^{\beta}+\frac{1}{n^{\alpha+\beta+1}}\left(\left|u_{n} v_{0}\right|+\left|u_{0} v_{n}\right|\right) \\
= & M_{1} M_{2} \sum_{k=1}^{n-1} \frac{1}{n}\left(1-\frac{k}{n}\right)^{\alpha}\left(\frac{k}{n}\right)^{\beta} \\
& +\frac{1}{n^{\alpha+\beta+1}}\left(\left|u_{n} v_{0}\right|+\left|u_{0} v_{n}\right|\right) \rightarrow M_{1} M_{2} \int_{0}^{1}(1-x)^{\alpha} x^{\beta} d x
\end{aligned}
$$

for $n \rightarrow \infty$.

Setting $u_{k}=(\mu+1)_{k} / k !=O\left(k^{\mu}\right)$ and $v_{k}=(\mu+1)_{k}(\lambda)_{k} / k ! k !=O\left(k^{\mu+\lambda-1}\right)$, Lemma 1 gives $\beta_{n}^{\lambda, \mu}=O\left(n^{2 \mu+\lambda}\right)$. Therefore, $\lim _{n \rightarrow \infty}\left(F_{n}^{\lambda, \mu}\right) \sim(1)=1$ holds, and according to [4, Proposition 1.3.10], we have the following result:

Theorem 2. Let $\mu \geq 0, \lambda \geq 1$; further, let $X$ denote $L^{p}(T), 1 \leq p<\infty$, or $C(T)$. Then for all $f \in X$

$$
\lim _{n \rightarrow \infty}\left\|F_{n}^{\lambda, \mu} * f-f\right\|_{X}=0
$$

is valid.

The rate of convergence for $F_{n}^{\lambda, \mu}$ can also be derived by means of Lemma 1. Setting

$$
u_{k}=\frac{(\mu+1)_{k}}{k !}=O\left(k^{\mu}\right)
$$

and

$$
v_{k}=\frac{(\mu+1)_{k}(\lambda)_{k}}{k ! k !}\left(1-\frac{k(k+\lambda)}{(k+1)(k+\lambda-1)}\right)=O\left(k^{\mu+\lambda-3}\right),
$$

we obtain

$$
\beta_{n}^{\lambda, \mu}-\beta_{n}^{\lambda, \mu}\left(F_{n}^{\lambda, \mu}\right)^{\sim}(1)=O\left(n^{2 \mu+\lambda-2}\right),
$$

provided $\mu+\lambda>2$. Since $\beta_{n}^{\lambda, \mu}=O\left(n^{2 \mu+\lambda}\right)$, we get

$$
1-\left(F_{n}^{\lambda, \mu}\right)^{-}(1)=O\left(n^{-2}\right),
$$

if $\mu+\lambda>2$. Thus (see [4, Propositions 1.5.11, 1.5.12]) we get

Corollary 4. Let $\mu \geq 0, \lambda \geq 1$, and $\mu+\lambda>2$. The positive trigonometric polynomials $F_{n}^{\lambda, \mu}$ define a Zygmund approximation sequence. In particular, we have

$$
\lim _{n \rightarrow \infty}\left\|F_{n}^{\lambda, \mu_{*}} f-f\right\|_{X}=O\left(n^{-\alpha}\right)
$$

for all $f \in \operatorname{Lip}^{*}(X, \alpha)$. 


\section{REFERENCES}

1. M. Abramowitz and I. A. Stegun, Handbook of mathematical functions, Dover Publ., New York, 1972.

2. R. Askey, Jacobi summability, J. Approx. Theory 5 (1972), 387-392.

3. __ Orthogonal polynomials and special functions, SIAM, Philadelphia, PA, 1975.

4. P L. Butzer and R. J. Nessel, Fourier analysis and approximation. I, Birkhäuser, Basel, 1971.

5. G. Gasper, Positive sums of the classical orthogonal polynomials, SIAM J. Math. Anal. 8 (1977), 423-447.

6. E. M. Stein and A. Zygmund, On the boundary behavior of harmonic functions, Orthogonal Expansions and Continuous Analogues (D. Haimo, ed.), Southern Illinois Univ. Press, Carbondale, IL, 1968, pp. 127-141.

7. G. Szegö, Orthogonal polynomials, Amer. Math. Soc., New York, 1951.

GSF-MÜNCHEN, MEDIS-INSTITUT, 8042 NeUHERBERG, GERMANY 